\title{
Construcción de un Modelo de Gestión como herramienta de sostenibilidad social, económica del "Complejo Turístico Dique de San Isidro", en la parroquia rural San Isidro, Cantón Morona
}

\author{
Construction of a Management Model as a tool to achieve the social, \\ economic sustainability of the "Complejo Turístico Dique de San Isidro", in \\ the rural parish of San Isidro, Canton Morona
}

\begin{abstract}
Tania Patricia Lara Cárdenas. ${ }^{1}$, Julio Cesar Benavides Lara. ${ }^{2}$ \& Alexis Jamil Cabrera Arteaga. ${ }^{3}$
\end{abstract}

Abstract. $\quad$ DOI: $\underline{\text { https://doi.org/10.33262/visionariodigital.v4i3.1298 }}$

The objective of the study is the construction of a MANAGEMENT MODEL for the project "Tourist Complex Dique San Isidro" investment of the Rural Parish Government San Isidro belonging to the Morona canton. The research necessarily had to alert social actors about the positive and negative impacts of tourism activity through a theoretical and conceptual framework adapted to local reality so that with this information decisionmakers have sufficient arguments for generating public value and social sustainability.

For the construction of the Management Model methodologically, the execution of three stages is proposed, which are based on the problems identified for the operation of the Complex, and then from the MAP OF KEY PROCESSES add the Process diagrams, input for the preparation of manuals; in addition to a Value Chain, an instrument for the articulation and creation of the tourist destination. Finally, a Management Scheme is inserted, supported by the Deming cycle and the Organizational and Functional Structures respecting the dynamics and legality.

For the third stage of the study, a DASHBOARD is proposed, which contains 129 management indicators distributed by key processes, frequency and managers. Undoubtedly, this tool must be socialized with all the actors and, of course, it must be contextualized in the territory to then recognize the usefulness for monitoring and evaluating the impact of the implementation and operation of the Dique de San Isidro Tourist Complex; as well as the generation of inputs for accountability.

\footnotetext{
${ }^{1}$ Escuela Superior Politécnica de Chimborazo, Extensión Morona Santiago -Ecuador. taniapatricia_laracardenas@hotmail.com.

2 Escuela Superior Politécnica de Chimborazo, Chimborazo Ecuador, jbenavides@espoch.edu.ec

${ }^{3}$ Presidente del del gobierno autónomo parroquial de San Isidro, alexisca3@gmail.com
} 
Keywords: Impacts of Tourism, Management Model for Tourist Resorts, Sustainability Tool.

\section{Resumen.}

El objetivo del estudio es la construcción de un MODELO DE GESTIÓN para el proyecto "Complejo Turístico Dique San Isidro" inversión del Gobierno Parroquial Rural San Isidro perteneciente al cantón Morona. La investigación tuvo que necesariamente alertar a los actores sociales acerca de los impactos positivos y negativos de la actividad turística a través de un marco teórico y conceptual adaptado a la realidad local para que con esta información los tomadores de decisiones tengan argumentos suficientes para la generación de valor público y sostenibilidad social.

Para la construcción del Modelo de Gestión metodológicamente se plantea la ejecución de tres etapas las que se fundamentan en los problemas identificados para la operación del Complejo para posteriormente a partir del MAPA DE PROCESOS CLAVES adicionar los diagramas de Procesos, insumo para la elaboración de manuales; además de una Cadena de Valor, instrumento para la articulación y creación del destino turístico. Finalmente se inserta un Esquema de Gestión apoyado en el ciclo de Deming y la Estructuras Organizacional y Funcional respetando la dinámica y legalidad.

Para la tercera etapa del estudio se propone un CUADRO DE MANDO, el que contiene 129 indicadores de gestión distribuidos por procesos claves, frecuencia y responsables. Sin duda que esta herramienta debe ser socializada con todos los actores y por su puesto debe ser contextualizada en territorio para reconocer entonces la utilidad para las acciones de monitoreo y evaluación del impacto de la implantación y funcionamiento del Complejo Turístico Dique de San Isidro; así como la generación de insumos para la rendición de cuentas.

Palabras clave: Impactos del Turismo, Modelo de Gestión para Complejos Turísticos, Herramienta de Sostenibilidad

\section{Introducción.}

La parroquia de San Isidro, según la información del Plan de Desarrollo y Ordenamiento Territorial 2015-2019 (PDOT, 165) es el resultado de varios procesos migratorios especialmente de indígenas de la provincia de Chimborazo; concentrándose especialmente en sectores: Puente Hondo, Totoras, Chanalá y Chillán. Para la década de 1960 llegan a este sector personas de origen colombiano cuyo objetivo era comercializar los productos como la cascarilla, el caucho, la canela.

El sector en donde actualmente se asienta la parroquia era conocida como "La Planada", para 1950 el padre salesiano Isidoro Formagio la funda con el nombre de San Isidro, 
posteriormente la parroquia rural fue creada mediante acuerdo ministerial el 6 de septiembre 1967 (Registro Oficial $N^{\circ}$ 206). Políticamente está conformada por su cabecera parroquial y cuatro barrios: San José-La Primavera-Occidental-La Unión; la comunidad del Edén y el caserío de Nueva Jerusalén. La parroquia de acuerdo a las proyecciones para el año 2015 cuenta con una población de 802 habitantes de las cuales $50,6 \%$ pertenecen al género femenino y el $49,4 \%$ al género masculino, con una tasa de crecimiento del 3,97\%. El 72\% se autoidentifica como mestizo y el $11 \%$ como indígena (INEC, 2010).

Para efectos de esta investigación "La Construcción del Modelo de Gestión como herramienta de sostenibilidad social, económica del "Complejo Turístico Dique de San Isidro" es el espacio público que contará con la infraestructura (edificación, accesos, servicios, conectividad) necesarios para ser considerado como un atractivo turístico cantonal. La implementación de este tipo de proyectos responde a las iniciativas para el desarrollo local y con el propósito de apoyar con la solución de la problemática social para el manejo del tiempo libre, recreación; así como aportar para el cambio de la matriz productiva en vista de las consecuencias de las malas prácticas agrícolas y la pobre calidad del suelo que a mediano plazo incrementarán tanto la frontera agrícola y procesos migratorios (campo-ciudad).

Este tipo de inversiones se justifican plenamente por el ejercicio de las competencias concurrentes del Gobierno Autónomo Descentralizado Parroquial de San Isidro y exclusivas de los Gobiernos Cantonal y Provincial (COOTAD, 2010) que promueven el fomento productivo y la adecuada gestión del producto turístico . La implementación del Complejo Turístico iniciativa de desarrollo sostenible tiene prevista una inversión de alrededor de 600 mil dólares con el financiamiento de los Gobiernos Provincial Municipal, Parroquial y el Banco de Desarrollo del Ecuador -EP- para la construcción de áreas de recreación, hospedaje y alimentación sobre la base del recurso agua y una construcción rustica a manera de dique que puede causar problemas tanto por la contaminación por la ausencia de servicios básicos como por la ausencia de normas técnicas. El complejo ocupará alrededor de $30.000 \mathrm{mts} 2$ con un enfoque sostenible que pretende "posicionar como atractivo turístico a nivel cantonal” (HARO, 2019).

Metodológicamente se trazó una secuencia didáctica y pedagógica con la ayuda de ilustraciones para que todos los actores sociales sin importar el nivel de formación comprendan el alcance de cada etapa. Se inicia con levantamiento de un marco conceptual sobre los impactos del turismo en economías de subsistencia y con la revisión del proyecto elaborado por el Gobierno Parroquial, lamentablemente el proyecto no especifica qué pasará con funcionamiento u operación del mismo. Por lo tanto, el estudio plantea la ejecución de tres etapas: Aproximación y contextualización de la actividad turística, Construcción del modelo de gestión y la elaboración de un cuadro de mando que incluye los indicadores de gestión para cada proceso clave. 
Una vez terminadas las tres etapas estamos seguros que la propuesta de MODELO DE GESTIÓN aportará a la consecución de los objetivos y por garantizar la sostenibilidad. Es de vital importancia apoyar este tipo de inversiones, para justamente antes de su operación se reconozca el papel gerencial de los funcionarios públicos y de esta manera superar la visión excesivamente centrada en la administración pasando a fortalecer la visión gerencial de los bienes públicos como lo refieren los autores Barzelay, 1998; Metcalfe y Richards, 1993. Uno de los ejes fundamentales de la nueva administración pública consiste precisamente el encontrar el mayor valor posible para los ciudadanos En la siguiente ilustración se puede apreciar la magnitud de la inversión.

\section{Metodología.}

Para el cumplimiento de los objetivos del estudio metodológicamente se plantea la ejecución de tres etapas, las que a continuación se describen:

I. Aproximación teórica y contextualización del impacto de la actividad turística. Con el objetivo de recabar y analizar las opiniones y criterios de diversos actores acerca del cambio de actividades (agricultura por turismo) en una población vulnerable y los niveles de complejidad para construir la cadena de valor del producto turístico con una adecuada articulación para la creación del destino turístico cantonal.

II. Construcción del modelo de gestión. El que se constituye en la herramienta didáctica para la administración del Complejo Turístico y aportes en la generación de políticas públicas que garanticen la sostenibilidad social y financiera de la inversión.

III. Elaboración de un cuadro de mando. Como complemento del modelo de gestión, se presentan una serie de Indicadores de Gestión, útiles para procesos de monitoreo, evaluación y activación de los procesos de mejora de la calidad.

\section{Resultados.}

\section{Etapa I}

Aproximación teórica y contextualización del impacto de la actividad turística.

La Organización Mundial de Turismo -OMT - define el turismo sostenible como la actividad económica que enfatiza en la satisfacción de las necesidades de los turistas, destinos de acogida actuales, y al mismo tiempo protege y mejora las oportunidades para el futuro (OMT, 2001). Las iniciativas de desarrollo basadas en el turismo tienen como metas la generación de empleo, el incrementado de divisas y la redistribución de los ingresos. Esta última meta es de vital importancia puesto que el dinero generado en el sitio o destino tiene esta característica de quedarse directamente en el productor del 
servicio al contrario de las actividades basadas en la exportación que tienen que atravesar por largos procesos para su obtención.

El principal problema en este tipo de iniciativas es el desconocimiento conceptual por parte de autoridades y actores sociales acerca del tipo de turismo que se desea implementar. La Parroquia Rural de San Isidro pretende construir un complejo turístico, vale la pena destacar que el autor Fabio Cárdenas en su texto de proyectos define: "El complejo turístico consta de conformaciones poco frecuentes porque depende de la existencia de uno o más atractivos de la más alta jerarquía, cuya visita, junto a la de otros que los complementan, implican una permanencia de tres días, o a veces más." (Cárdenas, 2006, pág. 27). Entonces surgen una serie de condiciones o requisitos para construir e implementar un complejo turístico, partiendo de la necesidad del inventario de atractivos turísticos (competencia del GAD Municipal), la articulación con otros atractivos y los más importante que se menciona el primer indicador de gestión del turismo que es el número de días de permanencia (\# días permanencia), el que para efectos de planificación se lo denomina índice de ocupación hotelera (IOH), el que determina en alguna medida la calidad del destino turístico.

Por ejemplo, si el cantón registra en su catastro 1000 plazas (camas hoteleras) y durante el año (360 días) ocupan 900 plazas tendrá un IOH del 90\% demostrando que existe alta demanda del destino y direcciona a contar con más plazas hoteleras para el próximo año. Por el contrario, sí en el mismo número de camas se ocupan al año 100 plazas obtendrá un $\mathrm{IOH}$ del $10 \%$, probablemente este destino desaparezca en el corto tiempo argumentando también el ciclo de vida de cualquier producto (Inicio-DesarrolloSaturación-Casi Desaparición). Este dato es fundamental, por lo tanto, es imperioso que se profundice el Estudio de Mercado y se identifiquen claramente los perfiles de los consumidores y lograr determinar los días de estancia.

Por otro lado, este tipo de turismo es utilizado como alternativa de desarrollo local que permite a un destino turístico alcance beneficios concretos como: mejorar su competitividad, aumentar y mantener la satisfacción de los turistas, incrementar y mantener la generación de ingresos y de empleo en la localidad, valorar las riquezas culturales y ambientales. Por lo tanto, si un determinado lugar -atractivo/destino- va a implementar un proyecto turístico es socialmente ético valorar los impactos negativos del turismo, sobre todo para que los rectores de las políticas públicas y las autoridades locales analicen todos los elementos e impactos y tomen las mejores decisiones con argumentos. En este sentido el principal problema o impacto negativo como la refiere Boullon (2006) es el cambio de actividad primaria del lugar o destino.

Sí la economía de la población está basada en la agricultura para la subsistencia y se propone a los servicios turísticos (Alojamiento, Alimentación, Recreación) como la nueva fuente de ingresos, es probable que se produzca división en los residentes locales, la separación espacial de las áreas turísticas del resto del centro turístico o en los alrededores del campo conlleva al aparecimiento de clases sociales, es decir se evidencia mejoras en 
las condiciones de vida solamente en aquella población que trabaja directamente en turismo y se ahonda la pobreza en aquella población que no participa en el turismo, a pesar de que los recursos les pertenecen a todos.

El mismo autor describe que espacialmente hay desplazamientos de poblaciones; por ejemplo, edificaciones que fueron construidas para vivienda, se cambian para otros usos. Es decir, se busca satisfacer las necesidades de los visitantes (turistas) y la población originaria tendrá que buscar otro sitio; y, en la mayoría de los casos se ven obligados a vender a cualquier precio.

Por estas razones es importante que para la toma de decisiones se cuente con los argumentos necesarios para invertir en turismo y no en servicios básicos. Indudablemente que para evitar la subutilización de los recursos turísticos se hace urgente el proyecto, pero es preciso que las autoridades locales consideren que antes de la implementación medir los impactos del turismo en una población vulnerable.

Sin lugar a dudas son más los impactos positivos pero la estrategia debe ser incluyente y participativa, por sobre todo en la generación de capacidades colectivas y el fomento de la asociatividad para que toda la población sea la beneficiaria y se traduzca en el mejoramiento de la calidad y condiciones de vida. Finalmente, la construcción de un destino turístico a más de la socialización del conocimiento acerca del tipo de turismo y sus consecuencias (impactos) se hacen necesarias acciones concretas para articular con todos los actores y autoridades del cantón.

\section{Etapa II}

\section{Construcción del modelo de gestión}

El término modelo proviene del concepto italiano de modello, la palabra puede utilizarse en distintos ámbitos y con diversos significados. Aplicado al campo de las ciencias sociales, un modelo hace referencia al arquetipo que, por sus características idóneas, es susceptible de imitación o reproducción. También al esquema teórico de un sistema o de una realidad compleja.

El concepto de gestión, por su parte, proviene del latín gesĭo y hace referencia a la acción y al efecto de gestionar o de administrar. Se trata, por lo tanto, de la concreción de acciones conducentes al logro de un negocio o de un deseo cualquiera. La noción implica además acciones para gobernar, dirigir, ordenar, disponer u organizar. De esta forma, la gestión supone un conjunto de trámites que se llevan a cabo para resolver un asunto, concretar un proyecto o administrar una empresa u organización. Por lo tanto, un modelo de gestión es un esquema o marco de referencia para la administración de una entidad.

Los modelos de gestión pueden ser aplicados tanto en las empresas, negocios privados; así como en la administración pública. Esto quiere decir que los gobiernos tienen un modelo de gestión en el que se basan para desarrollar sus políticas y acciones, y con el 
cual pretenden alcanzar sus metas y objetivos. Es importante mencionar que la diferencia en la utilización del modelo de gestión en lo privado y lo público. Mientras las empresas tienen como objetivos la consecución de lucro/rentabilidad/utilidades, en lo público está de por medio el bienestar social de la población.

Este asunto ha acarreado históricamente graves inconvenientes en el sector público, principalmente porque se han dedicado a administrar-supervisar cosas y dejar de lado el verdadero impacto en la población. En otras palabras, no saben valorar o cuantificar el resultado de sus acciones e inversiones en la población (mejora en salud, educación, etc.) en la mayoría de los casos por la escasa formación de las autoridades y funcionarios; además de que las autoridades cumplen con periodos para los que fueron electos y también incide la carga ideológica. En el siguiente análisis se quiere establecer directivas para que las autoridades locales tengan alternativas para construir el valor público.

Propuesta de Modelo de Gestión para la operación del Complejo Turístico Dique San Isidro

\section{Objetivos.}

- Identificar la dinámica social y la problemática en la operación y funcionamiento del Complejo Turístico Dique de San Isidro

- Construir un MODELO DE GESTIÓN para el Complejo Turístico Dique de San Isidro, con orientación hacia la sostenibilidad social y económica

- Establecer cuadro INDICADORES de resultados de gestión para la ejecución de las actividades de monitoreo y evaluación

\section{Identificación de la dinámica local}

La parroquia rural de San Isidro desde su creación se caracteriza por tener una actividad económica basada en la agricultura para la subsistencia y la evidente ausencia de capacidades colectivas y recursos tecnológicos como para generar cambios en la matriz productiva. Los propietarios de las fincas en la mayoría de los casos son beneficiarios de la Ley de la Reforma Agraria de 1964 , la que permitía a los colonos mestizos acceder como mínimo 50 hectáreas de terrenos por familia.

El territorio de la parroquia ofrece un gran potencial para ser aprovechado para el desarrollo económico y social, puesto que de las 12.861 hectáreas que comprende su territorio, el 64\% (8.267) de su territorio tienen diversidad de cultivos. Las autoridades locales en su plan de desarrollo cuantifican en 1.268 Unidades de Producción Agrícola UPA- de éstas 953 se dedican a la producción de banano, 660 al cultivo de camote, papa china y 660 a la yuca. También es necesario resaltar los abundantes recursos naturales que posee y que están dispuestos para el aprovechamiento óptimo y sostenido. Las autoridades locales tienen el propósito de convertirse es un centro de producción y 
comercialización agro ganadera, con el desarrollo de la agricultura tecnificada, rentable y competitiva. (PDOT, 64, 78).

La actividad comercial y de servicios en la parroquia San Isidro es ejecutada por el $27 \%$ de la población y se caracteriza por la intermediación siendo su principal proveedor la cabecera cantonal Macas. Cuentan con 65 establecimientos económicos registrados. Los servicios de restaurantes, bares, y servicio de internet, cubre la demanda existente; sin embargo, es necesario mejorar su calidad.

Es preciso destacar que en la parroquia se ejecuta un proyecto de piscicultura con 30 pequeños productores el que podría en alguna medida convertirse en un atractivo turístico y fortalecer la construcción del destino turístico cantonal.

\section{Problemas en el funcionamiento y operación del complejo turístico.}

Como resultado de la débil articulación y la ausencia de capacidades colectivas para apoyar la concreción de la visión de futuro de las autoridades en lo referente al mejoramiento de los procesos de producción e industrialización, la parroquia presenta varios problemas como el incremento de la frontera agrícola, contaminación del suelo, baja coberturas de servicios básicos, baja cobertura de la escolaridad.

Estos problemas que desde la perspectiva de desarrollo serían las prioridades para solucionarles hacen contrapeso con las potencialidades y la evidente subutilización de su paisaje cultural y el aprovechamiento del recurso agua y otros recursos naturales visibilizan la construcción del complejo turístico como estrategia de desarrollo local.

Tomando en cuenta que el proyecto se encuentra en fase de búsqueda del financiamiento se identifican los problemas en el funcionamiento del complejo, descritos a manera de hipótesis de trabajo tomando en cuenta que en la mayoría de los casos las operaciones de este tipo de complejos responden a la combinación de actividades recreativas (buen uso del tiempo libre) y la hostelería que incluyen los servicios de alimentos y bebidas. A continuación, se describen los principales problemas que se presentarían en el Complejo Turístico:

1. Desconocimiento de los perfiles de consumidor (visitantes y turistas)

2. Ausencia de cálculo de costos de producción sobre la base de la demanda real y los productos identificados.

3. No se tiene el registro de prestadores de servicios turísticos a nivel cantonal

4. Se carece de documentos y "resolución" para la formalización y activación de los procesos de selección y contratación de personal

5. Ausencia de protocolos para selección y capacitación del personal de todas las áreas de los servicios del complejo 
6. Se carece de estrategias para capacitar al personal (convenios de cooperación con las Universidades)

\section{Construcción del Modelo de Gestión}

Tomando en cuenta que conceptualmente no es lo mismo gestión y administración, sobre todo porque históricamente en administración pública se tiende a comparar con la gerencia de una empresa privada solamente en lo que a jerarquía (poder) y no por su rol en la ejecución el ciclo administrativo para la obtención de los resultados; por lo tanto, el modelo de gestión direcciona las acciones para gobernar, dirigir, ordenar, disponer $\mathrm{u}$ organizar.

Entonces se comprenderá que la gestión como el conjunto de actividades planificadas que se llevan a cabo para resolver un asunto, concretar una meta u objetivo institucional; por lo tanto, este modelo es un esquema o marco de referencia para la administración del Complejo Turístico Dique de San Isidro.

Para la construcción del modelo en mención, metodológicamente se inició contextualizando los conceptos acerca de los impactos del turismo en poblaciones que tienen como base económica y social actividades de subsistencia (agricultura) y carecen de procesos industriales o generadores de valor agregado, en este caso la parroquia de San Isidro.

En la siguiente ilustración 2 se describe el proceso metodológico adoptado por los autores para la construcción del modelo de gestión:

Figura 1. Proceso metodológico para la construcción del modelo de gestión.

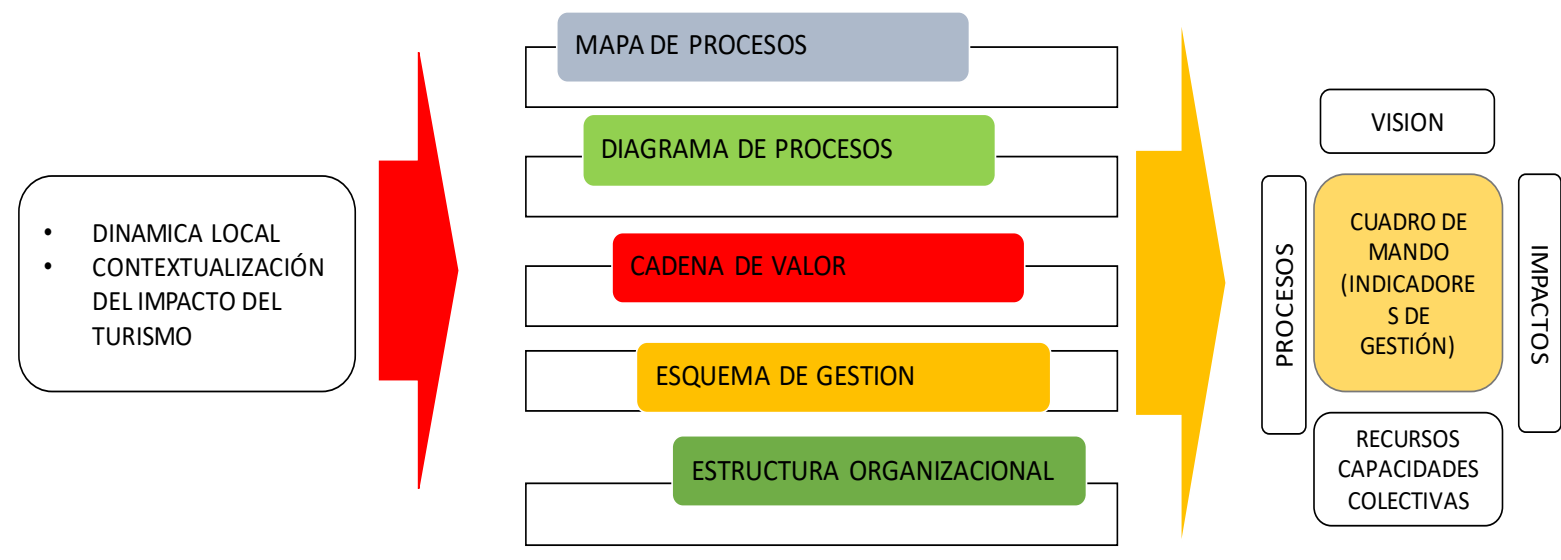

Fuente: Elaboración propia. 
Tratando de mantener una lógica comprensible para los futuros usuarios (internos y externos) y con la adecuada didáctica mediante el uso de ilustraciones se identifica un Mapa de Procesos, posteriormente se determinan los Diagramas de procesos, los que servirán de insumos para la elaboración de manuales de funcionamiento, para luego proponer una Cadena de valor del complejo instrumento necesario para que todos los actores comprendan que la adecuada articulación de cada los componentes, unidades y productos serán los que posicionen o permitan ser considerados como destino turístico.

Finalmente el modelo inserta las condiciones del Esquema de gestión visibilizando el ciclo de Deming con los productos de cada fase y la estructura organizacional que ha respetado la dinámica y legalidad de los gobiernos parroquiales rurales en el Ecuador. El modelo propuesto se complementa con la construcción de un cuadro de mando, definido así porque a cada proceso identificado se ha colocado los indicadores de gestión, útiles para realizar las acciones de monitoreo y evaluación del impacto de la implantación y funcionamiento del Complejo Turístico Dique de San Isidro.

\section{A continuación de describen a cada componente del modelo:}

\section{Mapa de procesos}

Con los marcos conceptual y referencial establecidos, sumado a la problemática para la operación y funcionamiento identificados, el modelo de gestión para el proyecto se inicia con la identificación de los procesos claves del Complejo. Es preciso recalcar que el Complejo está en etapa de búsqueda de financiamiento.

En este sentido la propuesta se constituye en la primera herramienta con la que cuenten las autoridades y que pueden tranquilamente anexar al proyecto como valor agregado; pues las entidades financieras visibilizarán fuertes argumentos sobre el impacto de la inversión en el desarrollo local. El hilo conductor del modelo como se había mencionado se basa en la identificación de procesos claves graficados en el mapa respectivo (ver ilustración 3).

Para el Complejo Turístico Dique San Isidro se identifican Procesos Estratégicos conocidos como procesos gerenciales de Dirección, Administración y Evaluación. Además de los Procesos claves u operativos que sirven para obtener el producto o servicio que se entrega al cliente mediante la transformación física de recursos y los Procesos de soporte: tienen como misión contribuir a mejorar la eficacia de los procesos operativos.

Es de vital importancia que se entienda como usuario no solamente al turista o visitante, se debe considerar como usuarios también a todos los actores y funcionarios que en alguna medida intervienen en la producción de los servicios. En la ilustración 3 en la siguiente página se pueden apreciar los procesos claves en color celeste en medio de los procesos estratégicos y de soporte los que intervienen y se correlacionan en la operación para cerrar el proceso de satisfacción de las necesidades y expectativas de los usuarios internos y externos del Complejo Turístico. 
Figura 2. Mapa de Procesos Complejo Turístico Dique de San Isidro

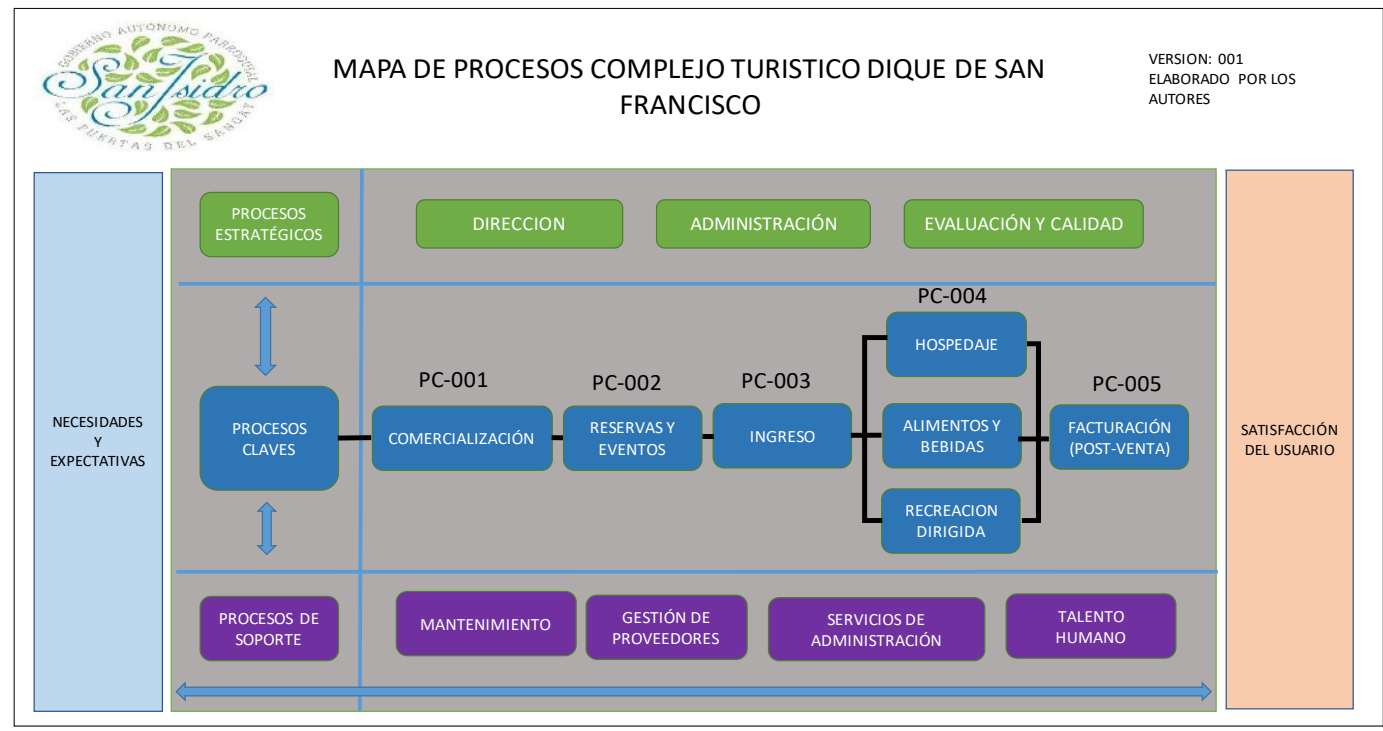

Fuente: Elaboración propia.

\section{PC-001: Comercialización}

Descripción: Este proceso en alguna medida es el que activa todo al resto. Es el resultado de la venta de productos del Complejo; pero también del resto de los atractivos que conforman "el producto turístico del Cantón".

Tabla 1. Resultado de la venta de productos del Complejo

\begin{tabular}{|c|c|c|c|}
\hline Entradas (input) & Acciones/subprocesos & Productos (output) & Indicadores \\
\hline $\begin{array}{c}\text { - Estrategia } \\
\text { Cantonal de } \\
\text { comercialización } \\
\text { - Metas de ventas } \\
\text { - Costos de } \\
\text { producción } \\
\text { - Canales de } \\
\text { comercialización } \\
\text { - Cronogramas }\end{array}$ & $\begin{array}{c}\text { - } \\
\text { Listado de reservas } \\
\text { - } \quad \text { Proyección de } \\
\text { ventas } \\
\text { - } \quad \text { Confirmación de } \\
\text { venta } \\
\text { - Ordenes de } \\
\text { producción }\end{array}$ & $\begin{array}{cc}\text { - } & \text { Catálogo de } \\
\text { productos } \\
\text { - } & \text { Precios de venta } \\
\text { - } & \text { Reportes } \\
\text { - } & \text { Informes } \\
\text { - } & \text { Base de datos } \\
\text { ventas } \\
\text { - } & \text { Evaluación post- } \\
\text { venta }\end{array}$ & $\begin{array}{c}\text { - } \begin{array}{c}\% \text { de ventas } \\
\text { efectivas }\end{array} \\
\text { - } \quad \% \text { cumplimiento } \\
\text { de Metas } \\
\text { - } \% \text { de repitencia } \\
\text { de consumo } \\
\text { - } \quad \text { Ventas por } \\
\text { producto } \\
\text { - \# de informes } \\
\text { generados } \\
\text { - Índice de } \\
\text { ocupación } \\
\text { hotelera }\end{array}$ \\
\hline
\end{tabular}

Fuente: Elaboración propia

PC002: Reservas y eventos

Descripción: La gestión de esta información es de vital importancia puesto que permitirá la planificación de los gastos y costos de producción. Es decir que el equipo de 
coordinadores debe tener el detalle del total de reservas (confirmadas) para iniciar la producción. Es importante destacar que una de las características del producto turístico es la de que se produce y consume en el mismo momento, es decir que el usuario o turista debe desplazarse efectivamente al lugar en donde se produce.

Tabla 2. Planificación de los gastos y costos de producción

\begin{tabular}{|c|c|c|c|}
\hline Entradas (input) & Acciones/subprocesos & Productos (output) & Indicadores \\
\hline $\begin{array}{cc}- & \text { Metas } \\
\text { - } & \text { Cronogramas } \\
\text { - } & \text { Presupuesto } \\
\text { - } & \text { Documento con } \\
\text { la confirmación } \\
\text { de reservas y } \\
\text { ventas } \\
\text { - } \quad \text { Talento } \\
\text { humano } \\
\text { disponible }\end{array}$ & $\begin{array}{cc}\text { - } & \text { Re-Confirmación } \\
\text { de venta } \\
\text { - } & \text { Compras e } \\
\text { insumos } \\
\text { - } & \text { Solicitud de } \\
& \text { personal }\end{array}$ & $\begin{array}{c}\text { - } \\
\text { Identificación de } \\
\text { perfiles de } \\
\text { clientes } \\
\text { - Ordenes de } \\
\text { producción } \\
\text { - Informe } \\
\text { ejecución } \\
\text { presupuestaria } \\
\text { - Ubicación y } \\
\text { distribución de } \\
\text { clientes }\end{array}$ & $\begin{array}{cc}\text { - } & \% \text { de reservas } \\
\text { efectivas } \\
\text { - } & \% \text { de reservas no } \\
& \text { efectivas } \\
- & \# \text { de eventos } \\
\text { realizados } \\
\text { - } & \text { Rotación de } \\
\text { personal } \\
\text { - } & \text { Quejas y Problemas } \\
\text { en la } \\
\text { comercialización } \\
\text { - Perfiles de clientes } \\
\text { identificados }\end{array}$ \\
\hline
\end{tabular}

Fuente: Elaboración propia.

\section{PC-004 Hospedaje/alimentos y bebidas/ recreación dirigida}

Descripción: Este proceso es la razón de ser del Complejo, es la tangibilización de los servicios. Todas las acciones realizadas desde la comercialización han dado su resultado, con la visita efectiva del usuario se hace necesario evidenciar las promesas de una buena comida, una habitación confortable y el conjunto de actividades perfectamente coordinadas para satisfacer las necesidades y expectativas de los usuarios.

Tabla 3. Tangibilización de los servicios

\begin{tabular}{|c|c|c|c|}
\hline Entradas (input) & Acciones/subprocesos & Productos (output) & Indicadores \\
\hline $\begin{array}{ll}\text { - } & \text { Metas de ventas } \\
\text { - } & \text { Costos de } \\
\text { producción } \\
\text { - } & \text { Re-Confirmación de } \\
\text { venta } \\
\text { - } & \text { Ordenes de } \\
& \text { producción } \\
\text { - } & \text { Check-in } \\
& \text { /Activación de } \\
\text { servicios para } \\
\text { facturación }\end{array}$ & $\begin{array}{ll}\text { - } & \text { Planificación } \\
& \text { compras } \\
- & \text { Asignación de } \\
& \text { personal } \\
- & \begin{array}{l}\text { Producción de los } \\
\text { servicios }\end{array} \\
- & \text { Control de calidad } \\
- & \text { Recreación activa } \\
& \text { y pasiva }\end{array}$ & $\begin{array}{ll}- & \text { Programa de } \\
& \text { recreación } \\
- & \text { Controles de } \\
& \text { consumo } \\
\text { - } & \text { Seguridad } \\
& \text { alimentaria } \\
- & \text { Supervisión } \\
- & \text { Reportes y registro } \\
& \text { para facturación } \\
& \text { (Check Out) }\end{array}$ & $\begin{array}{ll}\text { - } & \text { \% de uso de instalaciones } \\
\text { - } & \% \text { positivo de percepción } \\
& \text { del servicio } \\
\text { - } & \% \text { de satisfacción } \\
\text { - } & \# \text { de talleres de } \\
\text { capacitación } \\
\text { - } \quad \text { \# de reportes de } \\
\text { supervisión } \\
\text { - } \quad \text { \% rotación de personal } \\
\text { - } \quad \text { \# quejas y } \\
\text { recomendaciones } \\
\text { - de usuarios del programa } \\
\text { de recreación }\end{array}$ \\
\hline
\end{tabular}

Fuente: Elaboración propia. 


\section{PC-005 Facturación}

Descripción: Este proceso es el receptor de todos los procesos desde el registro de ingreso del usuario, es de fundamental importancia que se cuente con un sistema de facturación que de facilidades

\section{Diagramas de procesos}

Un diagrama de procesos facilita la concepción de la organización como un sistema. Existe una serie de símbolos estándar que representan diversas acciones en el mapa. Estos símbolos están normalizados por la American National Standards Institute (ANSI). En cuanto a las líneas, las continuas indican flujos fijos de información con transferencias directas de la información generada.

Debido a la característica del Complejo (en fase de financiamiento) se recomienda que los diagramas sean elaborados cuando el personal sea contratado y capacitado. Sin embargo, en la ilustración 4 cada coordinador deberá secuenciar las actividades y tareas para cada proceso. Esta información será de vital importancia en la construcción de flujogramas funcionales, manuales de procedimientos e informes.

Figura 3. Secuencia de desagregación de subprocesos Complejo Turístico Dique de San Isidro

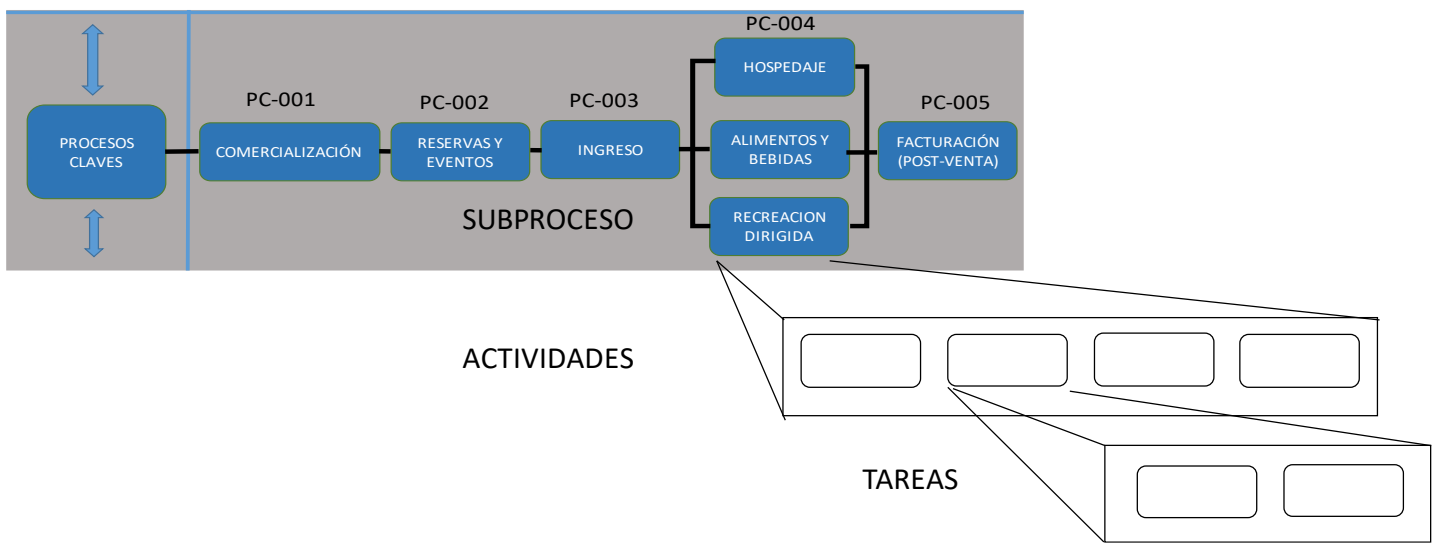

Fuente: Harrington H. James, Mejoramiento de los procesos de la empresa, McGrawHill, 1992

\section{Determinación de la "Cadena de valor" para el Complejo Turístico Dique San Isidro}

Para definir la cadena de valor de una empresa (pública o privada) se debe iniciar con la cadena genérica, las actividades de valor individuales se identifican en el Complejo. Cada categoría genérica puede dividirse en actividades discretas. La Mejora Continua, implica entender y trabajar en la cadena de valor. En este caso se parte de que el Complejo cuenta con una infraestructura turística envidiable la que junto con las políticas de dirección tendientes a la completa satisfacción de necesidades y exceptivas de los usuarios cuentan 
con el personal que está capacitado para la innovación del servicio y demostrar alta calidad en la preparación y ejecución de éstos.

En la Ilustración 5 se identifica a los componentes de la cadena de valor que tienen como base a los procesos de soporte y comercialización, el que se convierte en el proceso clave ya que de sus resultados depende la activación de todos los procesos.

Figura 4. Cadena de Valor Complejo Turístico Dique de San Isidro

\begin{tabular}{|c|c|}
\hline INFRAESTRUCTURA \\
\hline \hline DIRECCIONY POLITICAS DE TALENTO HUMANO \\
\hline INNOVACION \\
\hline REALIZACIÓN \\
\hline MANTENIMIENTO & $\begin{array}{c}\text { GESTIÓN DE } \\
\text { PROVEEDORES }\end{array}$ \\
\hline COMERCIALIZACIÓN \\
ADMINISTRACIÓN
\end{tabular}

Fuente: Elaboración propia.

\section{Esquema del sistema de gestión}

Los resultados de la implementación del sistema de gestión basado en el ciclo de Deming, permitirá conseguir la mejora integral de la competitividad, productos, servicios y como consecuencia se logrará la optimización de la productividad y el incremento en la participación del mercado para garantizar la rentabilidad social y financiera planteada.

El siguiente esquema permite visualizar las etapas de un plan de trabajo que debe seguirse para implementar un sistema de gestión. La organización es el punto de origen y destino puesto que debe haber involucramiento de todos los miembros del equipo de trabajo para alcanzar las metas con eficiencia, eficacia y calidad.

Figura 5. Sistema de Gestión Complejo Turístico Dique de San Isidro

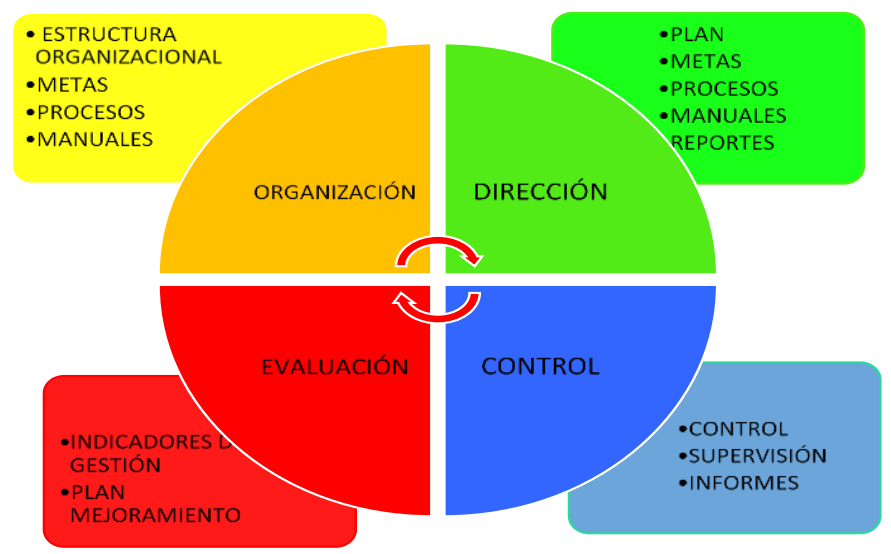

Fuente: Elaboración propia 


\section{Estructura organizacional}

De acuerdo a las características de la inversión para la construcción e implementación del Complejo Turístico Dique San Isidro se propone una estructura organizacional orientada al logro de metas y objetivos. Se describe a tres niveles jerárquicos (Dirección/Jefatura/Operativo). Es preciso destacar que esta estructura guarda coherencia en primer lugar con las disposiciones del COOTAD en el artículo 66 y con la cadena de valor que identifica los procesos estratégicos, claves y operativos los que promueven la generación de valor agregado de los productos puestos a disposición a los usuarios. Para darle operatividad la estructura es necesario que el Gobierno Parroquial establezca vía resolución y posterior ordenanza la creación de una empresa pública parroquial en concordancia al artículo 67 literal h del COOTAD. El la Ilustración 7 se puede apreciar la estructura propuesta.

Figura 6. Estructura Organizacional Complejo Turístico Dique de San Isidro

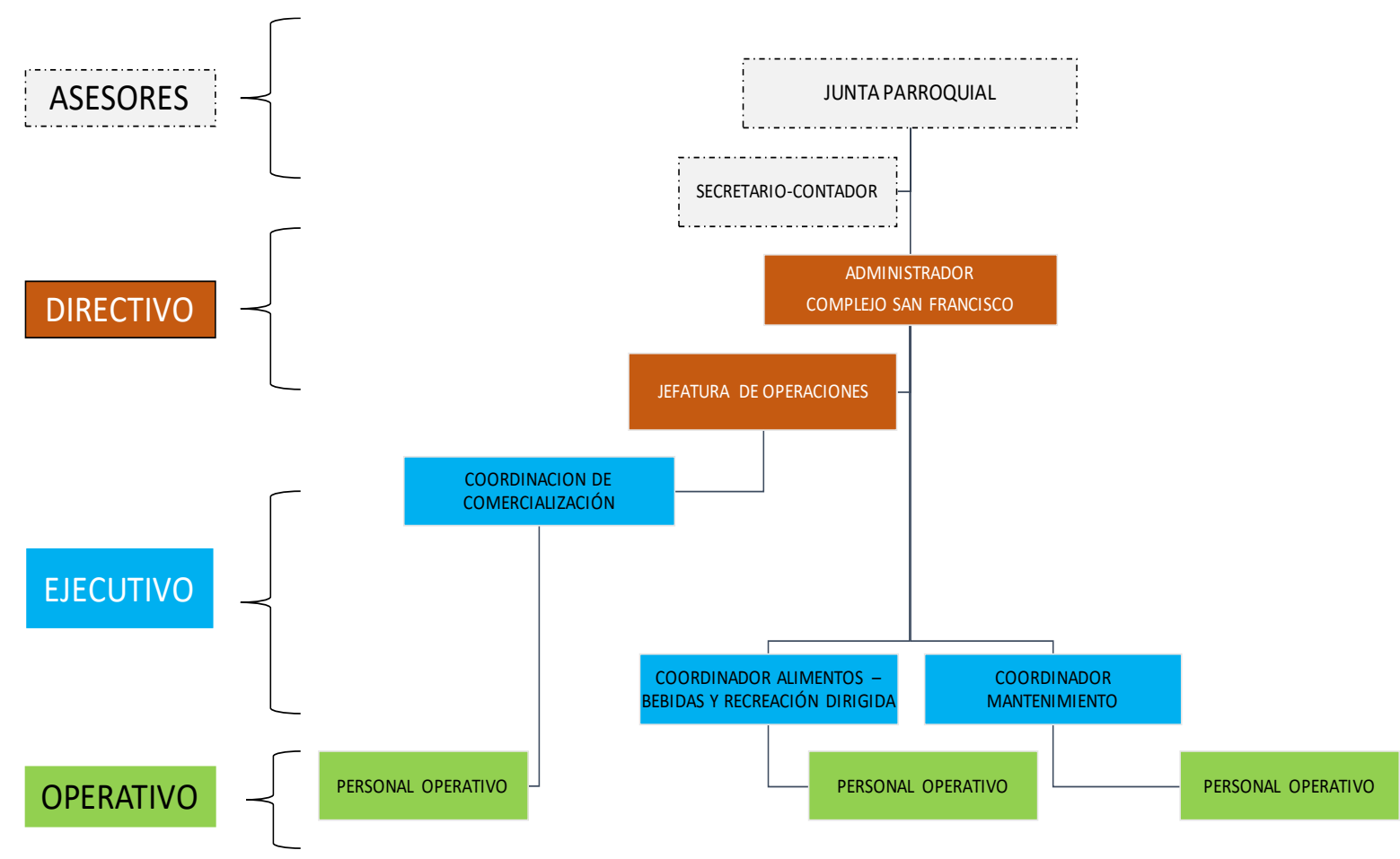

Fuente: Elaboración propia.

\section{Orgánico funcional}

El diseño organizacional es un proceso que determina las necesidades de la organización con el objetivo de alcanzar las metas establecidas a corto y mediano plazo. Es preciso dividir, asignar y reorganizar a partir de la cadena de valor y lograr la adecuada sincronización de procesos y conseguir los productos y resultados planteados. También es oportuno mencionar que esta herramienta es el insumo para la elaboración del cuadro de mando detallado en la tercera etapa de este estudio. En la siguiente matriz se 
determinan a manera de componentes los departamentos administrativos en función de las actividades que debe ejecutar el Complejo Turístico.

Tabla 4. Componentes los departamentos administrativos en función de las actividades que debe ejecutar el Complejo Turístico

\begin{tabular}{|c|c|c|c|}
\hline Niveles & Descripción & Funciones & Productos \\
\hline \multirow{2}{*}{$\begin{array}{l}\dot{0} \\
0 \\
0 \\
0 \\
0\end{array}$} & Junta parroquial /presidente & $\begin{array}{c}\text { - } \begin{array}{c}\text { Gestión del } \\
\text { desarrollo local }\end{array}\end{array}$ & \multirow{2}{*}{$\begin{array}{cc}\text { - } & \text { Construcción e } \\
\text { implementación de } \\
\text { políticas públicas } \\
-\quad \text { Rinden cuentas } \\
\end{array}$} \\
\hline & Secretario - contador & - Asesoramiento & \\
\hline \multirow{3}{*}{ 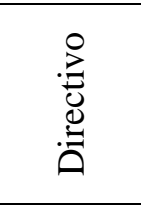 } & Administrador & $\begin{array}{c}\text { - Planificación } \\
\text { integral complejo }\end{array}$ & - Planifican \\
\hline & \multirow{2}{*}{ Jefe de operaciones } & - $\quad$ resoluciones & - Controlan \\
\hline & & - $\quad$ contrataciones & - $\quad$ Rinden cuentas \\
\hline \multirow{5}{*}{ 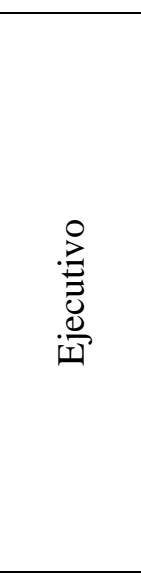 } & Coordinador comercialización & $\begin{array}{c}\text { - } \quad \text { Planificación y } \\
\text { ejecución de } \\
\text { procesos } \\
\text { habilitantes }\end{array}$ & - Ejecutan planes \\
\hline & Coordinador $\mathrm{a} \& \mathrm{~b}$ & $\begin{array}{c}\text { Elaboración de } \\
\text { formatos }\end{array}$ & - Evalúan \\
\hline & Coordinador mantenimiento & \multirow{3}{*}{$\begin{array}{c}\text { - } \quad \text { Evalúan los } \\
\text { resultados de los } \\
\text { procesos claves }\end{array}$} & - Reportes \\
\hline & Coordinador recreación & & - Informes \\
\hline & Personal operativo & & $\begin{array}{cc}\text { - } & \text { Formatos } \\
\text { evaluación } \\
\text { desempeño }\end{array}$ \\
\hline \multirow[b]{2}{*}{ 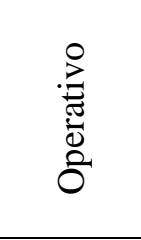 } & \multirow[b]{2}{*}{ Personal operativo } & - Aplican formatos & - Informes \\
\hline & & $\begin{array}{c}\text { Implementan y } \\
\text { gestionan control } \\
\text { de cumplimiento } \\
\text { de procesos }\end{array}$ & $\begin{array}{l}\text { - Aplican } \\
\text { evaluaciones de } \\
\text { desempeño }\end{array}$ \\
\hline
\end{tabular}

Fuente: Elaboración propia.

\section{Etapa III}

\section{Elaboración de un cuadro de mando.}

Esta herramienta es importante para la gestión puesto que proporciona un marco para ilustrar de qué modo la estrategia de comercialización del Complejo se articula con la creación de valor desde las perspectivas financiera, del cliente y del aprendizaje. Por otro lado, posibilita la detección de problemas que pueden ser resueltos con la prioridad del caso y no esperar al cierre del año fiscal (acciones de Monitoreo). 
De igual manera se generan alertas de procesos que necesitan estudios profundos y que acciones se pueden tomar para lograr el cumplimento de metas. Entonces contamos con un cuadro de mando para garantizar niveles óptimos de eficiencia, eficacia y efectividad. Para el efecto en la siguiente matriz a cada proceso clave se identifican los indicadores sobre la base de la unidad de medida y descripción (\% porcentaje), la frecuencia, el responsable para generar la información, medios de verificación (insumos para auditorías) y los productos esperados de cada proceso.

Tabla 5. Cuadro de mando para garantizar niveles óptimos de eficiencia, eficacia y efectividad.

\begin{tabular}{|c|c|c|c|c|c|c|c|c|c|c|c|c|}
\hline \multirow[b]{2}{*}{$\begin{array}{l}\text { PROCES } \\
\text { O CLAVE }\end{array}$} & \multirow[b]{2}{*}{ INDICADORES } & \multicolumn{5}{|c|}{ FRECUENCIA } & \multicolumn{4}{|c|}{$\begin{array}{l}\text { RESPONSABL } \\
\text { ES }\end{array}$} & \multirow[b]{2}{*}{$\begin{array}{c}\text { FUENTES } \\
\text { Y MEDIOS } \\
\text { DE } \\
\text { VERIFICA } \\
\text { CION }\end{array}$} & \multirow[b]{2}{*}{$\begin{array}{l}\text { PRODUCTOS } \\
\text { (OUTPUT) }\end{array}$} \\
\hline & & $\frac{\square}{\frac{D}{D}}$ & 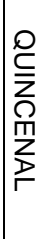 & 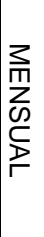 & 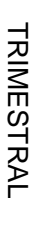 & 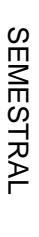 & 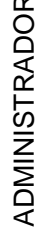 & 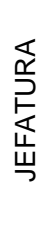 & $\begin{array}{l}\text { OD } \\
\frac{0}{\pi} \\
\frac{\pi}{\alpha} \\
\frac{\pi}{0} \\
0\end{array}$ & 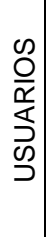 & & \\
\hline \multirow{7}{*}{$\begin{array}{c}\text { PC-001- } \\
\text { COMERCIA } \\
\text { LIZACION }\end{array}$} & - $\quad$ Ingresos por ventas $\mathrm{x}$ producto & $X$ & & & & & $\mathrm{O}$ & & & & \multirow{7}{*}{$\begin{array}{c}\text { Catálogo de } \\
\text { productos / } \\
\text { Informes/ } \\
\text { Registros }\end{array}$} & - Catálogo de productos \\
\hline & - $\quad \%$ de ventas efectivas & & & $\mathrm{X}$ & & & & $\mathrm{O}$ & & & & - $\quad$ Precios de venta \\
\hline & - $\quad \%$ cumplimiento de Metas & & & & $\mathrm{X}$ & & $\mathrm{O}$ & & & & & - Reportes \\
\hline & $\begin{array}{l}\text { - } \% \text { de repitencia de consumo } \\
\text { /Ocupación Hotelera }\end{array}$ & & & $\mathrm{X}$ & & & & $\mathrm{O}$ & $\mathrm{O}$ & $\mathrm{O}$ & & - Informes \\
\hline & - $\quad$ Ventas por producto & & & $X$ & & & $\mathrm{O}$ & $\mathrm{O}$ & & & & - $\quad$ Base de datos ventas \\
\hline & $\begin{array}{l}\text { - \# Perfiles de usuarios } \\
\text { identificados }\end{array}$ & & & & & $\mathrm{X}$ & $\mathrm{O}$ & $\mathrm{O}$ & $\mathrm{O}$ & $\mathrm{O}$ & & - Perfiles \\
\hline & - $\quad$ \# de informes generados & & $X$ & & & & $\mathrm{O}$ & $\mathrm{O}$ & $\mathrm{O}$ & & & - Evaluación post-venta \\
\hline \multirow{6}{*}{$\begin{array}{c}\text { PC-002- } \\
\text { RESERVAS } \\
\text { Y EVENTOS }\end{array}$} & - $\quad \%$ de reservas efectivas & $\mathrm{X}$ & & & & & & $\mathrm{O}$ & $\mathrm{O}$ & & \multirow{6}{*}{$\begin{array}{l}\text { Informes/Rep } \\
\quad \text { ortes/ } \\
\text { Formularios/C } \\
\text { ontratos/Presu } \\
\text { puestos }\end{array}$} & $\begin{array}{l}\text { Identificación de perfiles } \\
\text { de clientes }\end{array}$ \\
\hline & - $\quad \%$ de reservas no efectivas & $\mathrm{X}$ & & & & & & $\mathrm{O}$ & $\mathrm{O}$ & & & - $\quad$ Ordenes de producción \\
\hline & - $\quad$ \# de eventos realizados & & $X$ & & & & $\mathrm{O}$ & $\mathrm{O}$ & $\mathrm{O}$ & & & $\begin{array}{l}\text { - Informe ejecución } \\
\text { presupuestaria }\end{array}$ \\
\hline & - $\quad$ Rotación de personal & & & & $\mathrm{X}$ & & $\mathrm{O}$ & & $\mathrm{O}$ & & & \\
\hline & $\begin{array}{l}\text { - Quejas y Problemas en la } \\
\text { comercialización }\end{array}$ & $X$ & $\mathrm{X}$ & $\mathrm{X}$ & $X$ & & & $\mathrm{O}$ & $\mathrm{O}$ & $\mathrm{O}$ & & $\begin{array}{l}\text { - Ubicación y distribución } \\
\text { de clientes }\end{array}$ \\
\hline & - $\quad$ Perfiles de clientes identificados & & & & $\mathrm{X}$ & & $\mathrm{O}$ & $\mathrm{O}$ & $\mathrm{O}$ & & & \\
\hline \multirow{4}{*}{$\begin{array}{l}\text { PC-003- } \\
\text { INGRESO }\end{array}$} & - $\quad$ \# de visitantes ingresados & $X$ & & & & & & $\mathrm{O}$ & $\mathrm{O}$ & $\mathrm{O}$ & \multirow{4}{*}{$\begin{array}{l}\text { Facturas/Infor } \\
\text { mes }\end{array}$} & $\begin{array}{l}\text { Re-Confirmación de } \\
\text { órdenes de producción Catálogo } \\
\text { de productos }\end{array}$ \\
\hline & - $\quad \%$ de órdenes de trabajo activadas & $X$ & & & & & & $\mathrm{O}$ & $\mathrm{O}$ & $\mathrm{O}$ & & $\begin{array}{l}\text { Colocación de cintas de } \\
\text { identificación }\end{array}$ \\
\hline & - $\quad$ \# visitantes recibos y atendidos & $\mathrm{X}$ & & & & & & $\mathrm{O}$ & $\mathrm{O}$ & $\mathrm{O}$ & & - $\quad$ Reportes \\
\hline & - $\quad \%$ cumplimiento de Metas & & & $X$ & & & $\mathrm{O}$ & $\mathrm{O}$ & & & & - Informes \\
\hline \multirow{5}{*}{$\begin{array}{c}\text { PC-004 } \\
\text { HOSPEDAJE } \\
\text { /ALIMENTO } \\
\text { S Y } \\
\text { BEBIDAS/ } \\
\text { RECREACI } \\
\text { ÓN } \\
\text { DIRIGIDA }\end{array}$} & - $\quad \%$ de uso de instalaciones & $\mathrm{X}$ & $\mathrm{X}$ & $\mathrm{X}$ & $\mathrm{X}$ & $\mathrm{X}$ & & $\mathrm{O}$ & $\mathrm{O}$ & $\mathrm{O}$ & \multirow{5}{*}{$\begin{array}{l}\text { Registros/Ord } \\
\text { enes/Reportes/ } \\
\text { Formularios } \\
\text { aplicados }\end{array}$} & - $\quad$ Programa de recreación \\
\hline & $\begin{array}{l}\text { - } \% \text { positivo de percepción del } \\
\text { servicio }\end{array}$ & & & $X$ & $\mathrm{X}$ & $\mathrm{X}$ & & $\mathrm{O}$ & $\mathrm{O}$ & $\mathrm{O}$ & & - Controles de consumo \\
\hline & - $\quad \%$ de satisfacción & & & $X$ & $\mathrm{X}$ & $\mathrm{X}$ & & $\mathrm{O}$ & $\mathrm{O}$ & $\mathrm{O}$ & & - Seguridad alimentaria \\
\hline & - $\quad$ \# de talleres de capacitación & & & $X$ & & $\mathrm{X}$ & $\mathrm{O}$ & $\mathrm{O}$ & $\mathrm{O}$ & & & - Supervisión \\
\hline & - $\quad$ \# de reportes de supervisión & $\mathrm{X}$ & $\mathrm{X}$ & $\mathrm{X}$ & $\mathrm{X}$ & $\mathrm{X}$ & $\mathrm{O}$ & $\mathrm{O}$ & $\mathrm{O}$ & & & $\begin{array}{l}\text { - Reportes y registro para } \\
\text { facturación (Check Out) }\end{array}$ \\
\hline
\end{tabular}




\begin{tabular}{|c|c|c|c|c|c|c|c|c|c|c|c|c|}
\hline \multirow{4}{*}{$\begin{array}{l}\text { PROCES } \\
\text { O CLAVE }\end{array}$} & \multirow[b]{2}{*}{ INDICADORES } & \multicolumn{5}{|c|}{ FRECUENCIA } & \multicolumn{4}{|c|}{$\begin{array}{c}\text { RESPONSABL } \\
\text { ES } \\
\end{array}$} & \multirow{4}{*}{\begin{tabular}{|c} 
FUENTES \\
Y MEDIOS \\
DE \\
VERIFICA \\
CION \\
\end{tabular}} & \multirow{4}{*}{$\begin{array}{l}\text { PRODUCTOS } \\
\text { (OUTPUT) }\end{array}$} \\
\hline & & $\frac{\text { D }}{\frac{D}{D}}$ & 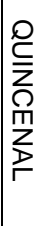 & $\begin{array}{l}3 \\
\frac{m}{2} \\
\frac{m}{2} \\
\risingdotseq\end{array}$ & 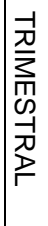 & 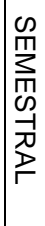 & 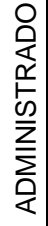 & 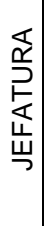 & 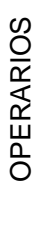 & 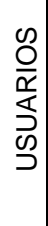 & & \\
\hline & - $\quad \%$ rotación de personal & & & & & $\mathrm{X}$ & $\mathrm{O}$ & $\mathrm{O}$ & & & & \\
\hline & - $\quad$ \# quejas y recomendaciones & & $\mathrm{X}$ & & X & $\mathrm{X}$ & $\mathrm{O}$ & $\mathrm{O}$ & $\mathrm{O}$ & $\mathrm{O}$ & & \\
\hline \multirow{5}{*}{$\begin{array}{c}\text { PC-004- } \\
\text { FACTURACI } \\
\text { ON }\end{array}$} & - $\quad$ Ingresos por ventas $\mathrm{x}$ producto & $\mathrm{X}$ & & & & & $\mathrm{O}$ & $\mathrm{O}$ & & & \multirow{5}{*}{\begin{tabular}{|c|c|} 
Registros/Fact \\
uras/Depósito \\
s/informes
\end{tabular}} & - Facturas \\
\hline & - $\quad \%$ cumplimiento de Metas & & $\mathrm{X}$ & & & & $\mathrm{O}$ & $\mathrm{O}$ & $\mathrm{O}$ & & & - Depósitos \\
\hline & - $\quad \%$ de repitencia de consumo & & & $\mathrm{X}$ & $\mathrm{X}$ & & $\mathrm{O}$ & $\mathrm{O}$ & $\mathrm{O}$ & & & Pagos \\
\hline & - $\quad$ Ventas por producto & $\mathrm{X}$ & $X$ & $\mathrm{X}$ & $\mathrm{X}$ & $\mathrm{X}$ & $\mathrm{O}$ & $\mathrm{O}$ & $\mathrm{O}$ & & & Informes \\
\hline & - $\quad$ Formas de pago & & $\mathrm{X}$ & $\mathrm{X}$ & $\mathrm{X}$ & $\mathrm{X}$ & $\mathrm{O}$ & $\mathrm{O}$ & & & & - $\quad$ Reportes \\
\hline
\end{tabular}

Fuente: Elaboración propia.

\section{Conclusiones:}

- El proyecto para la construcción e implementación del Complejo Turístico Dique de San Isidro patrocinado por el Gobierno Parroquial de San Isidro tiene limitaciones (subsanables) en su estudio de mercado, pues no identifica adecuadamente los perfiles de consumidores (usuarios) y tampoco especifica la etapa de implementación y operación del complejo. En este sentido el MODELO DE GESTIÓN propuesto pasa a llenar este vacío que puede ser decisivo al momento de la aprobación del crédito solicitado.

- En cumplimiento a la primera etapa del estudio, se logró construir un marco conceptual básico sobre los impactos del turismo en economías de subsistencia, positivos por la redistribución directa de los ingresos, generación de empleo, pero negativos por el cambio de actividades primarias, desplazamientos forzados y el aparecimiento de clases sociales que ahondan la desigualdad. Sobre la base de este marco las autoridades locales pueden iniciar procesos de sensibilización con toda la población para crear empatía y empoderamiento que se traduzca en la generación de valor público de la inversión y poder construir el destino turístico con argumentos basados en la ciencia.

- En vista de que el proyecto se encuentra en fase de financiamiento a manera de hipótesis se identifican los problemas que tendría su operación: Desconocimiento de los perfiles de consumidor (visitantes y turistas) Ausencia de cálculo de costos de producción sobre la base de la demanda real y los productos identificados. No cuenta con el registro de prestadores de servicios turísticos a nivel cantonal. Se carece de documentos y "resolución" para la formalización y activación de los procesos de selección y contratación de personal. Ausencia de protocolos para selección y capacitación del personal de todas las áreas de los servicios del 
complejo y no se cuenta con estrategias para capacitar al personal (convenios de cooperación con las Universidades)

- El modelo de gestión construido identifica un Mapa de Procesos, posteriormente se determinan los Diagramas de Procesos, los que servirán de insumos para la elaboración de manuales de funcionamiento. Se propone además la Cadena de Valor del Complejo instrumento necesario para que todos los actores comprendan que la adecuada articulación de cada los componentes, unidades y productos serán los que posicionen o permitan ser considerados como destino turístico. Finalmente se inserta un Esquema de Gestión visibilizando el ciclo de Deming con los productos de cada fase; así como las Estructuras Organizacional y Funcional respetando la dinámica y legalidad de los Gobiernos Parroquiales Rurales en el Ecuador en concordancia con COOTAD (2010).

- En la tercera etapa del estudio se construyó el CUADRO DE MANDO, el que contiene 129 indicadores de gestión distribuidos por procesos claves, frecuencia y responsables. Sin duda que esta herramienta debe ser socializada con todos los actores y por su puesto debe ser contextualizada en territorio para reconocer entonces la utilidad, y las acciones de monitoreo y evaluación del impacto de la implantación y funcionamiento del Complejo Turístico Dique de San Isidro; así como la generación de insumos para la rendición de cuentas.

\section{Referencias Bibliográficas:}

Banco Interamericano de Desarrollo, (2016). El Desarrollo y su Gerencia, $21^{\text {a }}$ edición. Notas de clase $\mathrm{N}^{\circ}$ 2. Efectividad en el desarrollo y la gerencia para resultados en el desarrollo América Latina. (http://creativecommons.org/licenses/byncnd/3.0/igo/legalcode)

Barzelay, M. \& Campbell, C. (2003). Preparing for the future (1st ed.). Washington, D.C.: Brookings Institution Press.

Biblioteca virtual de Derecho, E. y. (19 de 04 de 2020). https://www.eumed.net. Obtenido de https://www.eumed.net:https://www.eumed.net/libros-gratis/2007b/298/ley1964.htm

Boullon, C. (2006): Planificación del espacio turístico. México Trillas

Cárdenas F. (2006). Proyectos Turísticos: Localización e inversión (2da ed.) México. Trillas

Código Orgánico de Organización Territorial, Autonomía y Descentralización (2010). Quito. Asamblea Nacional

Flick, U. (1992). Introducción a la investigación cualitativa (1st ed., Vol. 1). Madrid: Morata.

Gurría di-Bella. (1991). Introducción al Turismo. México. Trillas

Gobierno Autónomo Descentralizado Municipal de Morona Santiago. (2016). Proyecto construcción de Complejo Turístico 
Haro, M. (2019). Proyecto Complejo Turístico San Isidro, Gobierno Autónomo Descentralizado Parroquial San Isidro

Harrington, J (1992). Mejoramiento de los procesos de la empresa, McGraw-Hill

INEC. (2010). Censo de Población y Vivienda, Quito

OMT. (2001). Organización Mundial de Turismo. Conceptos de Turismo Sostenible. Madrid, OMT

PDOT MORONA. (2019). Plan de Desarrollo y Ordenamiento Territorial Cantón Morona 2019-2021

PDOT SAN ISIDRO. (2019). Plan de Desarrollo y Ordenamiento Territorial 2019-2021

Robbins, S. \& Coulter, M. (2000). Administration (8th ed.). México: Prentice-Hall Hispanoamericana.

Rojas y Nacato. Técnica de Flujogramas. (1980). Quito, ESPE

Ripoll y Hernández, Graciela. Turismo Popular: Inversiones Rentables. (2002). México, Trillas 


\section{PARA CITAR EL ARTÍCULO INDEXADO.}

Benavides Lara, J. C., Lara Cárdenas, T. P., Oleas Carrillo, E. R., \& Murillo Pinos, M. C. (2020). Diagnóstico económico del criadero hacienda holstein Puculpala. Visionario Digital, 4(3), 102-121. https://doi.org/10.33262/visionariodigital.v4i3.1298

\section{Ciencia \\ LDigital}

El artículo que se publica es de exclusiva responsabilidad de los autores y no necesariamente reflejan el pensamiento de la Revista Visionario Digital.

El artículo queda en propiedad de la revista y, por tanto, su publicación parcial y/o total en otro medio tiene que ser autorizado por el director de la Revista Visionario Digital.
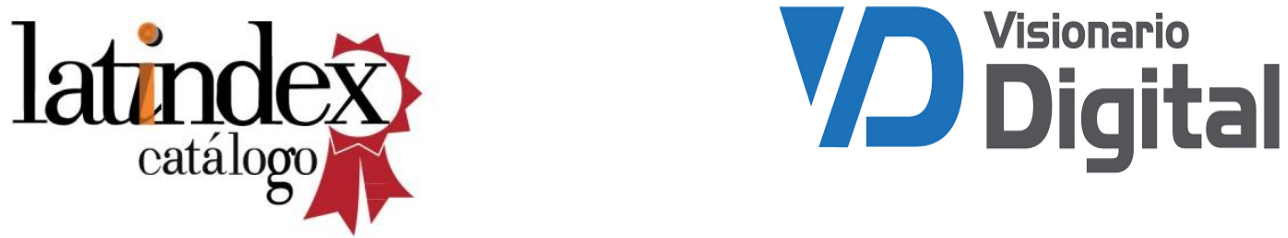